\title{
Two Negative Minima of the First Normal Stress Difference in a Cellulose-Based Cholesteric Liquid Crystal: Helix Uncoiling
}

\author{
Coro Echeverria, ${ }^{1}$ Pedro L. Almeida, ${ }^{1,2}$ Oscar F. Aguilar Gutierrez, ${ }^{3}$ Alejandro D. Rey, ${ }^{3}$ \\ Maria Helena Godinho' \\ ${ }^{1}$ I3N - CENIMAT, Faculdade de Ciências e Tecnologia, Departamento de Ciência dos Materiais, FCT/UNL, Caparica 2829-516, \\ Portugal \\ ${ }^{2}$ Area Departamental de Física, Instituto Superior de Engenharia de Lisboa, ISEL/IPL, R. Conselheiro Emídio Navarro, 1, Lisboa \\ 1959-007, Portugal \\ ${ }^{3}$ Department of Chemical Engineering, McGill University, Montréal, Québec, Canada \\ Correspondence to: C. Echeverria (E-mail: coro@fct.unl.pt) or A. D. Rey (E-mail: alejandro.rey@mcgill.ca) or M. H. Godinho \\ (E-mail:mhg@fct.unl.pt)
}

Received 27 September 2016; accepted 17 February 2017; published online 16 March 2017

DOI: $10.1002 /$ polb.24332

ABSTRACT: The shear rate dependence of material functions such as shear viscosity $(\eta)$ and the first normal stress difference $\left(N_{1}\right)$ were given and interpreted earlier by Kiss and Porter. Their widely accepted work revealed the possibility of having a negative minimum of $N_{1}$ for polymeric liquid crystals. In this work, we disclose for the first time the evidence of two negative $N_{1}$ minima on a sheared cellulosic lyotropic system. The lower shear rate minimum is ascribed to the uncoiling of the cholesteric helix, as theoretically predicted earlier. Our findings contribute also to the understanding of the other minimum already reported in the literature and attributed to the nematic director tumbling mode. Moreover, the elastic change that the
LC-HPC sample undergoes during the helix unwinding of the cholesteric structure is also by means of oscillatory measurements. This study is a contribution for the understanding of the structure-properties relationship linked with the complex rheological behavior of chiral nematic cellulose-based systems and may help to improve their further processing. (c) 2017 Wiley Periodicals, Inc. J. Polym. Sci., Part B: Polym. Phys. 2017, 55, 821-830

KEYWORDS: biopolymers; cellulose derivative; helix uncoiling; liquid-crystalline polymers (LCP); polymeric liquid crystal; rheology
INTRODUCTION The rheology of cellulosic systems has raised many issues and fundamental questions that are still unanswered. Considering the complexity of the Hydroxypropylcellulose (HPC) aqueous solution structure, a better insight into the interplay between flow and orientation could lead, for example, to improved processing strategies for the preparation of materials with controlled alignment, ${ }^{1}$ and to establish structure-properties relationships. Up to date, experimental observation and theoretical analysis put in evidence that the rheological response of polymeric liquid crystals (PLC) under shear flow is strongly affected by the type of liquid crystal (LC) phase present in the system and the structural transformations of the director field. ${ }^{2-5}$ Rheological studies on the HPC/water system have shown that, in the liquid crystalline phase, three distinct regions of steady shear flow can be observed $^{6,7}$ as first stated by Onogi and Asada. ${ }^{6-8}$ These regimes correspond to significantly different local system configurations resulting from the material response to the imposed shear stress. ${ }^{8}$
Besides the typical "three-regime" curve, characteristic of most PLC, another unusual property of some PLC is the existence of a shear rate range over which steady state first normal stress difference, $N_{1}$, is negative.

When shearing non-Newtonian fluids, the effect of shear rate ends up in the formation of viscous shear stress $\left(\sigma_{12}\right)$, and normal stress differences $\left(N_{1}=\sigma_{11}-\sigma_{22}, N_{2}=\sigma_{22}-\sigma_{33}\right)$, being 1 and 2 the shear direction and the perpendicular direction, and 3 the neutral direction. ${ }^{9,10}$ In isotropic polymers the magnitude of $N_{1}$ is zero or positive, but as mentioned and stated in the literature, this magnitude presents a shear rate dependent sign changing behavior, evolving from positive to negative and positive again at high shear rates. ${ }^{4,7,11-14}$

This evidence served as motivation for theoretical studies where the dynamics of the cholesteric structure of PLC under shear flow conditions were studied using continuum mechanics approach, as in the work by Rey. ${ }^{15,16}$ These

Additional Supporting Information may be found in the online version of this article.

(c) 2017 Wiley Periodicals, Inc.

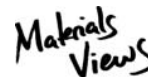


calculations suggested interesting physical phenomena resulting from the cholesteric structure interaction with the flow field during shear flow. ${ }^{3}$

Our experiments on sheared cholesteric liquid crystalline HPC demonstrate that the shear rate dependence of $N_{1}$ undergoes double negative minima of $N_{1}$ that correspond to the helix uncoiling and the non-equilibrium nematic structures of the LC, respectively. At low shear rates, the material does not align with the direction of the imposed shear, which is consistent with the soft response of the material and is often pictured as stacked polydomain. But when the applied shear increases, the polydomain structure begins to orient with the flow; the cholesteric helix unwinds giving rise to a complete alignment of the nematic director with the direction of the flow.

In addition, it is known that normal forces such as $N_{1}$ provide information regarding the stored elastic energy during flow. ${ }^{17}$ In this work, we are able to understand the phenomenology behind the first minimum of $N_{1}$ and detect, by means of oscillatory measurements, elastic changes that the LC-HPC sample undergoes during shear when the cholesteric helix unwinds.

Our study explores deeper on the origins of the nonequilibrium shear induced helix unwinding, exposing the presence of an additional negative minimum, which is interpreted as the complex helical unwinding and is sensitive to the material's shearing history. The alignment of the anisotropic structures that compose the PLC results in unique physical properties, thus the understanding of the shearinduced structural changes is paramount for further applications as seen for the case of cellulosic soft-motor recently developed. ${ }^{18,19}$ Therefore, the objective of this study is to identify experimentally the helix uncoiling that LC-HPC undergoes during shear flow.

\section{EXPERIMENTAL}

\section{Materials}

HPC with a molecular weight, $M_{\mathrm{w}}=100,000 \mathrm{Da}$ and $M_{\mathrm{S}}=3$ was purchased from Sigma-Aldrich and used as received. For the following studies, HPC was dissolved in water at $50 \mathrm{wt}$ $\%$. The prepared samples were sealed, kept in the dark to avoid light damage, and stirred every two days. A complete dissolution of HPC was obtained in 2 weeks exhibiting a cholesteric phase. ${ }^{20}$

\section{Methods}

Rheological characterization of the LC-HPC solutions was performed using a stress-controlled rheometer Bohlin Gemini HR nano, with a cone-plate $\left(20 \mathrm{~mm}\right.$ diameter and $2^{\circ}$ cone) for flow and oscillatory measurements. A solvent trap was used to avoid the solvent's evaporation.

Flow experiments were carried out as follows: (i) Shear rate ramp was performed between 0.01 and $500 \mathrm{~s}^{-1}$. (ii) Transient measurements were carried out for the following shear rates: $0.1,0.3,0.6,1,1.4,2,2.4,3,3.75,4.5,5,6,7,10,12$, $15,20,30,50,80$, and $100 \mathrm{~s}^{-1}$. All the tests were performed at $25{ }^{\circ} \mathrm{C}$. Both sets of experiments were preceded by $60 \mathrm{~s}$ of equilibration time prior to the tests.

For the dynamic measurements, frequency sweep tests were performed in a range of frequency from 0.1 to $50 \mathrm{~Hz}$. All the oscillatory measurements were carried out within the linear viscoelastic range (LVR) of the sample and at room temperature at a constant strain of $0.01 \%$. The LVR was determined by performing a strain sweep test at a non-destructive frequency of $1 \mathrm{~Hz}$.

Rheo-NMR experiments were carried out on a $(300 \mathrm{MHz})$ Bruker Avance III spectrometer. The ${ }^{2} \mathrm{H}-\mathrm{NMR}$ experiments were performed with a 7.049T superconducting magnet, corresponding to a deuterium resonance frequency of 46.072 $\mathrm{MHz}$, with a Couette-flow device and a saddle coil. ${ }^{20,21}$ The LC-HPC/ $\mathrm{D}_{2} \mathrm{O}$ sample was sheared at shear rates in the range of 0.1 to $100 \mathrm{~s}^{-1}$. The shear measurement was started with acquisition of DNMR spectra during time at desired constant shear rates. The relaxation process after cessation of the imposed shear was further monitored. Transverse relaxation times $T_{2}$ were determined using the Carr-Purcell-MeiboomGill (CPMG) sequence. The following parameters of the CPMG sequence were used: deuterium frequency resonance: $46.072 \mathrm{MHz}$; recycle delay: $5 \mathrm{~s}$; time to allow pulse ringdown: $50 \mu \mathrm{s}$; duration of FID: $300 \mu \mathrm{s}$ and pulse widths at $90^{\circ}$ and $180^{\circ}$ of 125 and $250 \mu \mathrm{s}$, respectively, both using a $\mathrm{RF}$ power of $50 \mathrm{~W}^{22}$

\section{RESULTS AND DISCUSSION}

As mentioned in the introduction, some LC polymers as LCHPC show a typical shear rate dependence of the viscosity describing a characteristic "three-regime" curve first indicated by Onogi and Asada. ${ }^{8}$ That first study put forward a widely accepted domain-based model that accounted for the shear rate dependence of the viscosity by correlating the response of polydomain nature LC in solution to shear flow. Three regions in the viscosity curve were distinguished and explained by Onogi and Asada $^{8}$ as follows: in Region I, the polydomain cholesteric structure is not altered at such low shear rates but domains flow over each other, resulting in the first shear-thinning region. The orientation of the initially unaffected polydomains starts to change due to the flow in Region II. This will be further discussed later in this section in terms of theoretical work of Rey ${ }^{15,16}$ where the helical axis is placed in the direction of the vorticity and the minimum is related to the onset of the wagging regime over the tumbling regime observed in Region I corresponding to low shear rate. Finally, Region III shows the second shear thinning of the curve, in which it is assumed that the alignment of the LC polymer is finally achieved.

In Figure 1, we show the viscosity (left $y$-axis) and normal force $\left(N_{1}\right)$ (right $y$-axis) as a function of the shear rate for the cholesteric liquid crystalline solution of $\mathrm{HPC}-\mathrm{H}_{2} \mathrm{O} 50 \mathrm{wt}$ $\%$. Two different shear thinning regions can be identified at 


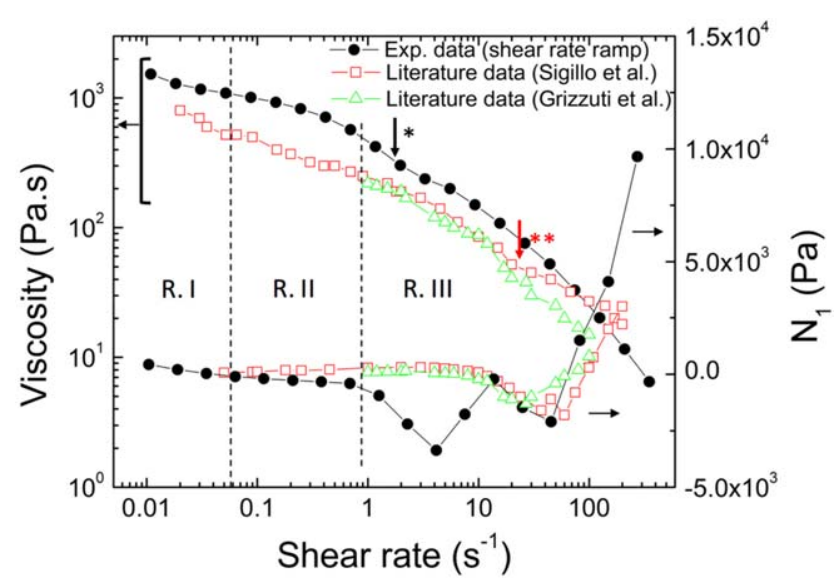

FIGURE 1 Evolution of both viscosity and first normal stress difference $\left(N_{1}\right)$ measured in a non-steady state mode (Full symbols). Empty red squares and green triangles correspond to flow curve of HPC/H2O 50 wt \% (100 kDa) extracted from Sigillo and Grizzuti, J Rheol (1978-present), 1994, 38, 589-599 and Grizzuti et al., Rheol Acta, 1993, 32, 218-226, reproduced by permission, respectively, in which 100 strain units were applied at each point. Black arrows* and red arrow** indicate the hesitation point of the viscosity shear dependence corresponding to experimental (black) and literature data (red)). [Color figure can be viewed at wileyonlinelibrary.com]

low and high shear rates representing Region I and Region II respectively, whereas Region II corresponds to the intermediate shear rate values separating the other regions. It is worth noting that the shear thinning occurring at low shear rates is less pronounced when compared to previously reported data. $6,14,23$ The results regarding the shear rate dependence of the first normal stress difference, $N_{1}$, present a novel behavior in the region between 1 and $100 \mathrm{~s}^{-1}$, where two negative minima at approximately $4 \mathrm{~s}^{-1}$ and $40 \mathrm{~s}^{-1}$ and an inflexion point at $15 \mathrm{~s}^{-1}$ are observed.

Up to now, first normal stress difference shear rate dependence was generally accepted to give rise to a single negative minimum of $N_{1}$, characteristic of LC polymers. ${ }^{8,24,25}$ A representative example of this behavior is shown in the results also represented in Figure 1 obtained by Sigillo et al. ${ }^{14}$ and Grizzuti et al. $^{23}$ for a $\mathrm{HPC} / \mathrm{H}_{2} \mathrm{O} 50$ wt \% sample $\left(M_{\mathrm{w}}=100\right.$ $\mathrm{kDa}$ ). Those results fit our data corresponding to the second minimum of $N_{1}$. But there is no precedent in the literature describing experimentally the presence of a lower shear rate $N_{1}$ minimum to the one just described. Additionally, this first minimum is coupled with the presence of a "kink" or hesitation point, ${ }^{26,27}$ which is a decrease of viscosity that is found for the shear rate at which $N_{1}$ decays (between 1 and $\left.2 s^{-1}\right)$.

Theoretical work done by Marrucci and Maffettone ${ }^{28}$ related the hesitation point (together with the decay of the first normal stress difference showing negative values) to a drastic change in the dynamics of the director. They considered that the material switches from a situation where the single domain never reaches a time-independent regime toward a stationary regime at higher shear rates, where the director aligns with the flow direction. However, in our experimental work (shear rate ramp) this hesitation point appears together with the onset of the first minimum of $N_{1}$ decay for lowest shear rate minimum and not at high shear rates.

It is important to highlight that the experiments carried out by Sigillo et al. ${ }^{14}$ and Grizzuti et al. ${ }^{23}$ were performed under steady state conditions after an application of 100 strain units, which is considered to be the minimum needed to achieve a steady state. In particular, Grizzuti et al. ${ }^{25}$ applied a protocol prior to each measurement which consisted in pre-shearing the sample at $1 \mathrm{~s}^{-1}$ during $600 \mathrm{~s}$ and then allowing it to rest for $600 \mathrm{~s}$ so that the recovery of the chiral structure occurs. However, in our previous study of LC-HPC under shear performed by Rheo-NMR, ${ }^{20}$ we found that the recovery of the HPC chiral structure in the relaxation process after shearing at $1 \mathrm{~s}^{-1}$ took more than $1000 \mathrm{~s}$ to occur. As this fact could be one of the reason why there is no precedent in the literature regarding the experimental detection of the helix uncoiling, this issue will be further discussed in the manuscript.

To confirm the presence of the first negative minimum of $N_{1}$ observed in the shear rate ramp, transient measurements were performed within the shear rate range of $0.1-100 \mathrm{~s}^{-1}$ as shown in Figure 2. As observed from Figure 2A, the evolution of the viscosity in time depict more stable curves at shear rates below $10 \mathrm{~s}^{-1}$ than those obtained for higher shear rates [Fig. 2(B)]. The time needed for the viscosity to achieve a steady state is indeed higher when LC-HPC is subjected to high shear rates $\left(>30 \mathrm{~s}^{-1}\right)$.

Similar to what is observed for the viscosity, the time needed for $N_{1}$ to achieve a steady state increases with the applied shear rate. However, it is remarkable that $N_{1}$ exhibits oscillation or damping behavior at the specific shear rates where $N_{1}$ undergoes its minimum value $\left[\sim 3 \mathrm{~s}^{-1}\right.$, see Fig. 2(A)] or at the decay of $N_{1}$ prior to the first minimum $\left(\sim 1-2.4 \mathrm{~s}^{-1}\right.$, see Supporting Information Figure S4).

It is worth mentioning that edge fracture flow instability can occur at high shear rates, as stated in the literature, as no mercury or silicon oil bath were used. ${ }^{6,23,29}$ Although we are aware that such flow instability could affect results obtained at such high shear rates, quantitatively accurate data are not essential for our purpose, as results regarding the second minimum of $N_{1}$ described in the Figure 1 were already studied and described in the literature. ${ }^{14,23}$ Besides, our main concern in this study is to experimentally detect the helix uncoiling through the observation of the first of the two minima of $N_{1}$.

We used transient measurements as a tool to build the flow curve of LC-HPC sample. From the plots shown in Figure 2, we collected data of viscosity and $N_{1}$ when the sample had been subjected to 150,200 , and 250 strain units $(\gamma=\dot{\gamma} t)$ for each shear rate (Fig. 3) and compared to the shear rate ramp and literature flow curve, already shown in Figure 1. 
A

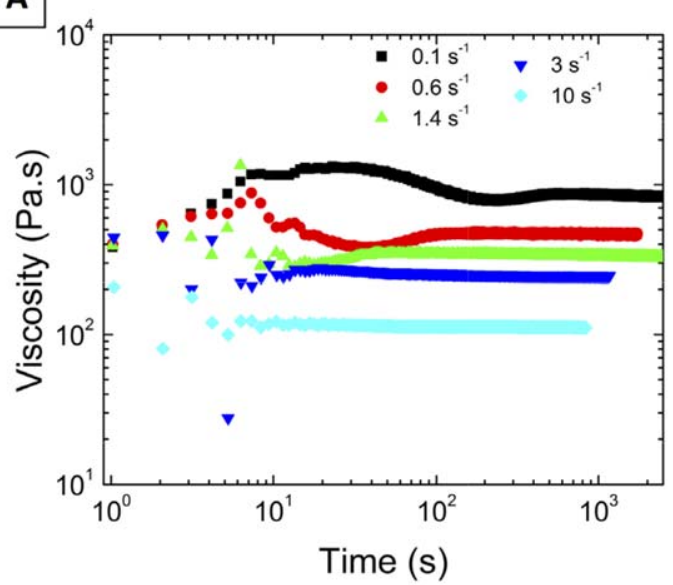

B

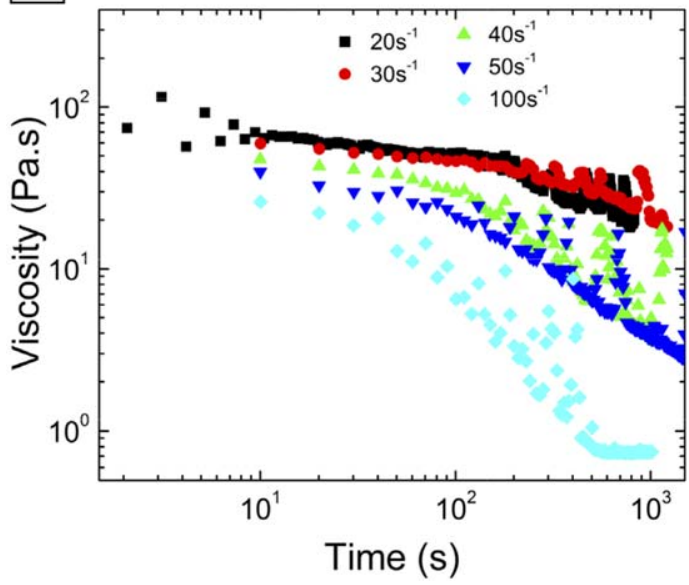

$A^{\prime}$

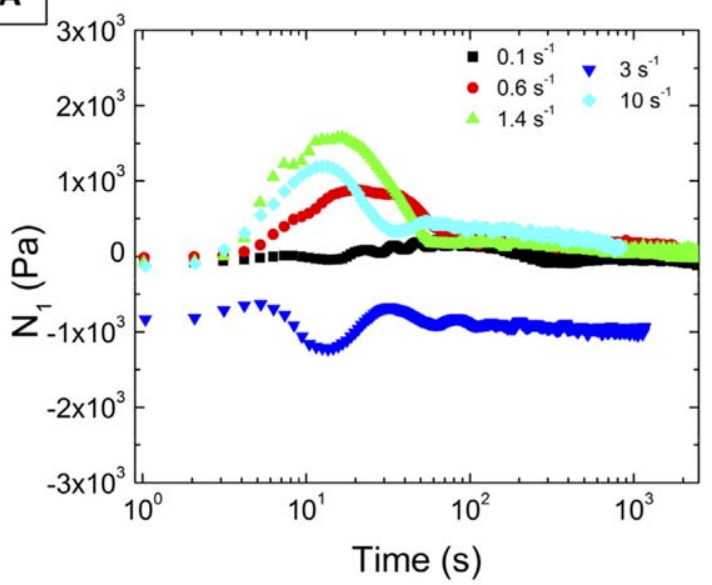

B'

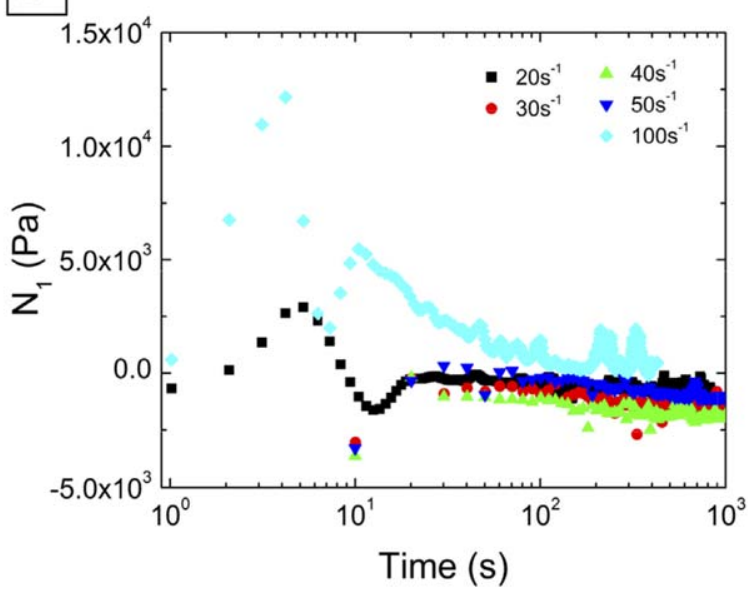

FIGURE 2 Representative transient measurements of LC-HPC (50 wt \%) measured at room temperature in which the evolution of viscosity $(\mathrm{A}, \mathrm{B})$ and $N_{1}\left(\mathrm{~A}^{\prime}, \mathrm{B}^{\prime}\right)$ with time when subjected to constant shear rates corresponding to Region II $\left(\mathrm{A}, \mathrm{A}^{\prime}\right)$ and Region III $\left(B, B^{\prime}\right)$ of the flow curve. (See also Supporting Information Fig. S4 and S5). [Color figure can be viewed at wileyonlinelibrary.com]

The viscosity shear-dependence shown in Figure 3 presents a similar behavior to that observed in literature and do not vary with the increase of strain units. However, if we focus on the transient measurements, it is observed that when the sample is subjected to shear rates above $20 \mathrm{~s}^{-1}$ [Fig. 2(C)], more time and thus more strain units are needed to achieve the steady state. Even the application of 250 strain units is not enough to reach the steady state above $20 \mathrm{~s}^{-1}$ for $N_{1}$. To minimize the error associated to the construction of this flow curve, we ensured that for each shear rate, the imposed shear was enough so that the strain units applied were sufficient to achieve a steady state. Thus, to obtain an accurate flow curve, we recorded the values of the viscosity and $N_{1}$ when the steady state of both parameters is reached independently and not at a defined strain unit, and represented them in Figure 4(A). The shear rate dependence of the viscosity shows similar results to that of the shear rate ramp depicted in Figure 2 as well as those found in the literature.

The results obtained for shear dependence of $N_{1}$ confirm the behavior already described by the non-steady-state shear rate ramp, in which two minima of $N_{1}$ at $\sim 4$ and $\sim 40 \mathrm{~s}^{-1}$ and an inflexion point at $\sim 15 \mathrm{~s}^{-1}$ are observed. The second minimum of $N_{1}$ appears at shear rate values $\left(\sim 40 \mathrm{~s}^{-1}\right)$ consistent with Region III of the flow curve, where the LC-HPC system is expected to be completely aligned. Moreover, the second minimum of $N_{1}$ corresponds to the wagging and further alignment of the nematic structure of the LC-HPC as described by Larson et al. ${ }^{11}$ and experimentally establish in the literature. ${ }^{14}$ But the minimum of $N_{1}$ found at $\sim 4 \mathrm{~s}^{-1}$ has not been experimentally observed before and could be symptomatic of a regime transition where the cholesteric structure is disturbed. In fact, we consider that it could be related to the uncoiling of the cholesteric helix, which has not been experimentally detected yet by standard rheology.

As a confirmation of the presence of this two negative minimum of $N_{1}$, a preliminary study of the evolution of the second normal stress difference $\left(N_{2}\right)$ with shear rate was also performed. The calculated $N_{2}$ values are represented together with $N_{1}$ in Figure 4(B) (see also section 4 of Supporting Information and Fig S6). As observed from the figure, the 

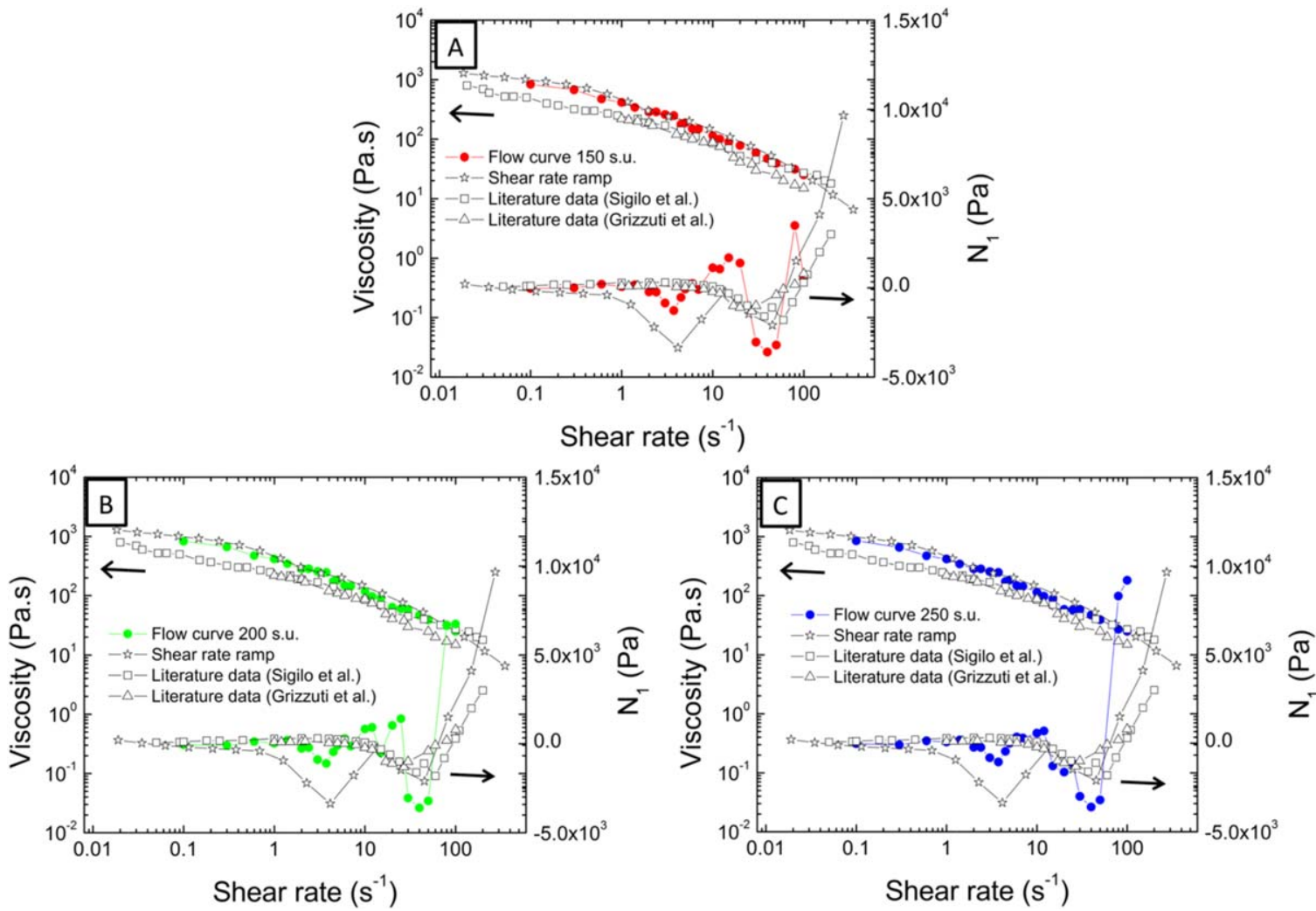

FIGURE 3 Evolution with shear rate of viscosity and $N_{1}$ when the sample is subjected to (A) 150 , (B) 200 , and (C) 250 strain units. For a better observation and as comparison, all graphs present the shear rate ramp shown in Figure 1 and the flow curve obtained by Sigillo and Grizzuti, J Rheol (1978-present), 1994, 38, 589-599 and Grizzuti et al., Rheol Acta, 1993, 32, 218-226, reproduced by permission for HPC/Water $50 \mathrm{wt} \%\left(M_{\mathrm{w}}=100 \mathrm{kDa}\right)$. [Color figure can be viewed at wileyonlinelibrary.com]

evolution of $N_{2}$ with shear rate also showed the two maxima already described for $N_{1}$, besides of the lower values of $N_{2}$ compared to $\left|N_{1}\right|$. These results could exclude the possibility that the observed first minimum of $N_{1}$ is a consequence of unsteady phenomena, indicating that the LC-HPC sample undergoes indeed two elasticity regime changes along the experiments
Changes in sign in $N_{1}$ correlate with the local dynamics of the average rod orientation which is a function of the Deborah number $(D=\tau \dot{\gamma} ; \tau$ : molecular relaxation time) and the shear-rate dependent tumbling $\lambda$ parameter. ${ }^{30}$ At low $D_{\mathrm{e}}$, when $<1$ the material is denoted tumbling or non-aligning and when $>1$ the material is of the aligning type and the director aligns at the Leslie angle
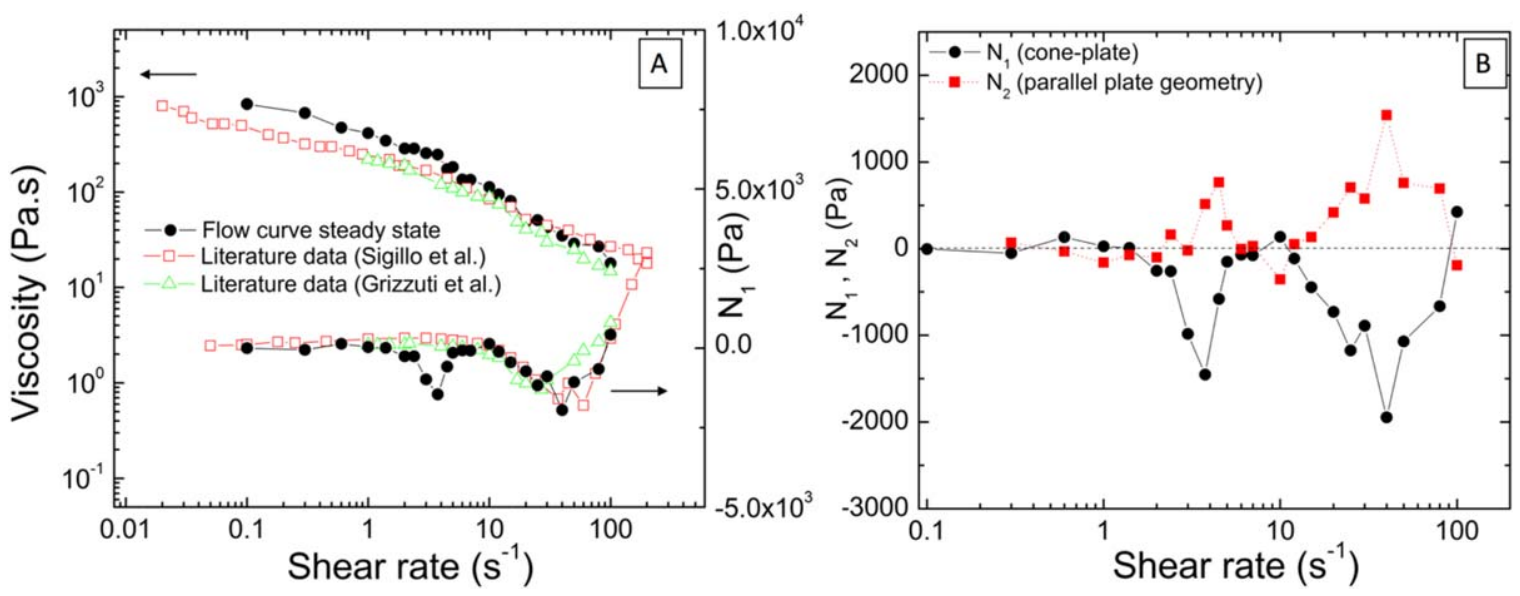

FIGURE 4 (A) Steady state flow curve obtained of data collected from transient measurements when sample reaches the steady state at each applied shear rate (full circles). For the sake of comparison, the flow curve obtained from literature (red square and green triangle $)^{14,23}$ are also plotted. (B) Evolution of $N_{1}$ and $N_{2}$ with shear rate for the shear rate regions where the two peaks emerge. (See also section 4 of Supporting Information and Figure S6). [Color figure can be viewed at wileyonlinelibrary.com] 


$$
\Theta_{L}=\frac{1}{2} \cos ^{-1} 1 \lambda \pm n \pi
$$

For non-aligning materials $\left(\lambda<1\right.$ at low $\left.D_{\mathrm{e}}\right)$, increasing shear rate introduces a cascade of orientation modes: tumbling (time periodic) \&cenveo_unknown_entity_wingdings_F0E0; wagging (time oscillatory) \&cenveo_unknown_entity_wingdings_F0E0; stationary (steady state). The first tumbling-to-wagging transition occurs when $N_{1}<0$ and the minimum in $N_{1}$ corresponds to the wagging mode. This flow-structure relation corresponds to chiral nematics.

For chiral nematics, previous work demonstrates that for shear flow-aligning material, a monodomain of a cholesteric whose chiral axis is aligned along the vorticity (normal to the shear plane) with no anchoring (no torques), helix uncoiling occurs when: ${ }^{15,31}$

$$
K=\gamma_{1}(\lambda-1) \dot{\gamma}
$$

where $K_{2}$ is the Frank twist constant, $P$ corresponds to the equilibrium undistorted pitch (usually in the micron range), $\gamma_{1}$ is the rotational viscosity and $\dot{\gamma}$ is the shear rate. The helix uncoiling is a non-equilibrium transition. Conversely, the same helix but of a tumbling material will not uncoil under weak shear because flow torques are always negative: $\gamma(\lambda-1) \dot{\gamma}<0$ as long as $\lambda<1$. In this latter case, under low $D_{\mathrm{e}}$, the cholesteric helix of a tumbling material rotates without changes in pitch. As $D_{\mathrm{e}}$ increases and the tumblingwagging transition is approached, it has been shown that $\lambda$ is not a fixed parameter but oscillates between a value lower than one $\lambda$ and a value larger than one $\lambda$. It has been shown that $\lambda$ increases with shear rate and it diverges at the tumbling \&cenveo_unknown_entity_wingdings_F0E0; wagging transition. Hence, we can see that even for a non-aligning material at low $D_{\mathrm{e}}$ such as HPC solutions, the helix uncoiling for an ideal vorticity-aligned helix can occur at some shear rate $\gamma$ because

$$
\frac{K_{2}}{P^{2}}=\gamma_{1}(\lambda-1) \dot{\gamma}_{\mathrm{c}}
$$

is now fulfilled because $\lambda>1$. In our case, the critical shear rate was estimated to be around $1.77 \mathrm{~s}^{-1}$ from the hesitation point of the viscosity following the end of the Newtonian plateau and corresponds also to the onset of the $N_{1}$ decay for lowest shear rate minimum. Using material property estimates $(K=5 \mathrm{pN}, P=10 \mu \mathrm{m})$ we find $\lambda=1+$ $\left(2.8 \times 10^{-2} / \gamma_{1}\right)$ which according to ${ }^{30}$ is in the tumbling mode and consistent with a $N_{1}<0$ if we are close to the tumbling-wagging transition. In experiments, we have a population of helix orientations, domains, defect nucleation and annihilation, so the model of helix uncoiling is semiquantitative.

\section{Rheo-NMR Analysis}

The Rheo-NMR technique combines rheology with NMR spectroscopy and has become a powerful tool to detect different systems' local configuration and orientation. In the study of LC-HPC/ $\mathrm{D}_{2} \mathrm{O}$ system, $\mathrm{D}_{2} \mathrm{O}$ acts as a probe, sensing the behavior of LC-HPC under shear.

Experimentally, an initial protocol is performed to ensure a reproducible initial state for every run. ${ }^{20-22}$ In this protocol, the LC-HPC solution subjected to a pre-shear, with a shear rate of $3 \mathrm{~s}^{-1}$ during $60 \mathrm{~s}$ and left resting during $1 \mathrm{~h}$ to get a stabilized NMR spectrum. After this procedure, all samples showed an initial central (single) peak, with no quadrupolar splitting, which is indicative of a disordered $\mathrm{D}_{2} \mathrm{O}$ environment. Thus, this spectrum corresponds to a polydomain LC solution of randomly oriented cholesteric domains. When applying shear to the system, deuterium spectra evolves toward peak splitting, behavior indicative of some degree of order acquired by the domains sensed by $\mathrm{D}_{2} \mathrm{O}$. (see Figure 5).

In this case, Rheo-NMR experiments were performed to better understand the effect of shear in the helix structure and in its further recovery. From the deuterium spectra collection obtained with time at different constant shear (0.1$100 \mathrm{~s}^{-1}$ ), ${ }^{20,22,32}$ it was possible to determine the maximum splitting attained at each shear rate and plotted with respect the applied shear [Fig. 6(A)]. The obtained results put in evidence a critical shear rate in the range of 15 to $20 \mathrm{~s}^{-1} .^{20}$ Above this value, the peak splitting becomes independent of the applied shear, indicating that the system has reach a maximum degree of order; alignment of the director is complete and independent of the shear. ${ }^{20,21}$ This critical shear rate was also detected in the flow curve describing the inflexion point between the two negative minima of $N_{1}$ (see Fig. 1). By combining the information obtained from both rheology and Rheo-NMR, it is possible to confirm that above $15-20 \mathrm{~s}^{-1}$ the pseudo-nematic state appears; the helix is uncoiled due to shear.

From the collection of deuterium spectra obtained with time after cessation of shear (relaxation process), it was possible to determine the minimum rest time necessary for the chiral structure to recover. Interestingly, two different trends are observed dependent on the previously applied shear as shown in Figure 6(B): (i) Relaxation after cessation of shear rate $>1 \mathrm{~s}^{-1}$ : The observed trend indicated that the relaxation time necessary for the chiral structure to recover decreases with the applied shear. (ii) Relaxation after cessation of shear rate $<1 \mathrm{~s}^{-1}$. In this case, the time necessary for the chiral structure to recover increases with shear rate. This different behavior confirms that the shear history affects the structure, and that the recovery time of the chiral structure is completely dependent on the previous induced deformation. It is noteworthy, that the maximum time required for the LC-HPC chiral structure to recover occurs when pre-sheared at shear rate close to $1 \mathrm{~s}^{-1}$.

In addition to that, evolution of the transverse relaxation time $\left(T_{2}\right)$ with time was determine at constant shear for the shear rate range below the critical shear rate, $15 \mathrm{~s}^{-1}$. The acquisition of the $T_{2}$ data was performed using specific delays between measurements during the shear and 

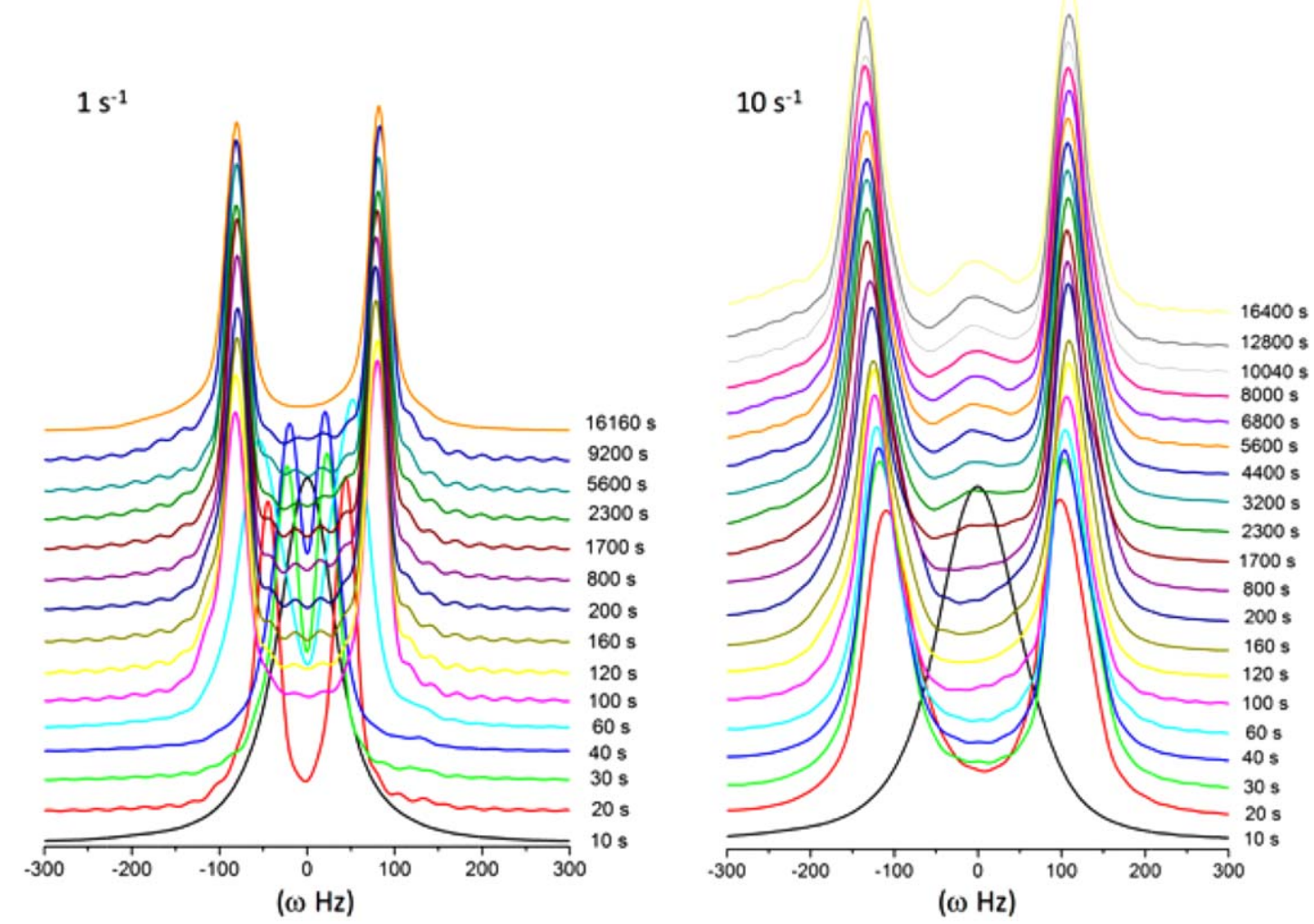

FIGURE 5 Representative deuterium spectra collection obtained with time for LC-HPC/D2O (50 wt \%) during shearing at constant shear rate of $1 \mathrm{~s}^{-1}$ and $10 \mathrm{~s}^{-1}$. [Color figure can be viewed at wileyonlinelibrary.com]

subsequent relaxation processes. The $T_{2}$ values were obtained from the fitting of the magnetization exponential decay obtained using a CPMG sequence and are representative of the LC-HPC behavior at a molecular level. As observed from Figure $6(\mathrm{C})$, the increase of the applied shear give rise to higher $T_{2}$ values, which reflects a less "solid-like" system. For a better observation, we extracted $T_{2}$ values in the stationary state [at $2000 \mathrm{~s}$ of Fig. 6(C)] and represented as a function of the applied shear, as shown in Figure 6(D). Interestingly here again two different $T_{2}$ trend are observed. From 0.1 to $1 \mathrm{~s}^{-1}$, an abrupt increase of $T_{2}$ is observed, which indicates a rapid evolution from a "solid-like" to a less "solid-like" behavior. Above $1 \mathrm{~s}^{-1}$, the slope of $T_{2}$ evolution with time is less pronounced, tending to the independency with respect to the applied shear This two differentiated relaxation processes, could also be indicative of a different local configuration under shear, which present the tendency change around $1 \mathrm{~s}^{-1}$.

Therefore, the results obtained from Rheo-NMR experiments highlighted two critical shear rates around $1 \mathrm{~s}^{-1}$, and 15$20 \mathrm{~s}^{-1}$, where relaxation process changes and the maximum degree of order is achieved, respectively. Interestingly, such critical shear rates are coincident with that observed in the steady state flow curve [see Fig. 4(A)]: (i) close to $1 \mathrm{~s}^{-1} N_{1}$ become negative, prior to the first minimum observed at 3$4 \mathrm{~s}^{-1}$ (ii) $15-20 \mathrm{~s}^{-1}$, that in Rheo-NMR measurements account for the shear rate value at which the maximum degree of order is achieved, in the steady state flow curve described the inflexion point between both negative minima of $N_{1}$.

To better describe the behavior of LC-HPC system under shear, we have collected in Table 1 the transitions observed in $N_{1}$, Rheo-NMR and theory, as a summary of the different regimes encountered in all shear rates of the three-regime curve.

\section{Dynamic Measurement}

As indicated by Marruci and Maffetone ${ }^{13}$ the application of shear stress into a rod-like LC polymer could result either in the modification of the director or it could induce a modification of the order parameter, which, in both cases, induces a negative value of the normal stress difference $\left(N_{1}\right)$. To understand the observed behavior of $N_{1}$, we considered the application of a pre-shear treatment to the LC-HPC sample to determine if the behavior of $N_{1}$ presented by LC-HPC could be affected by the induced different initial orientation over the system (see also section 1 of Supporting Information and Fig S1A).

For that purpose, we chose the shear rate at which the first minimum of $N_{1}$ is evident $\left(3.75 \mathrm{~s}^{-1}\right)$, and perform a preshear $\left(1 \mathrm{~s}^{-1}\right.$ during $60 \mathrm{~s}$ which correspond to 60 strain units) to induce changes in the structure prior to the following transient measurement at such critical shear rate 
A

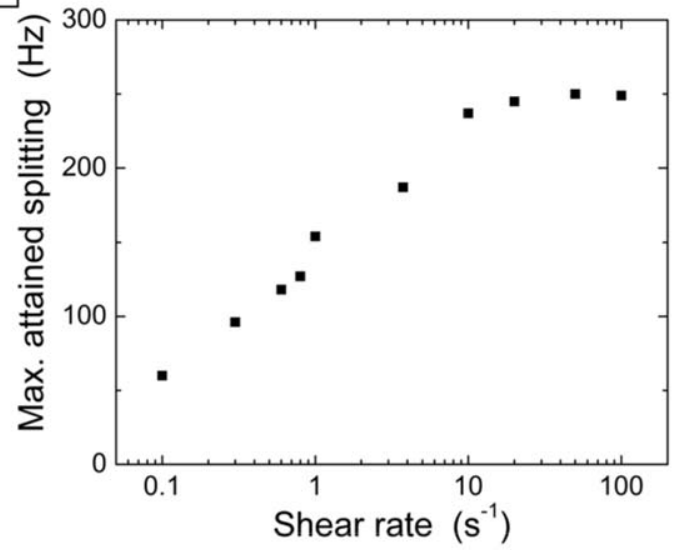

C

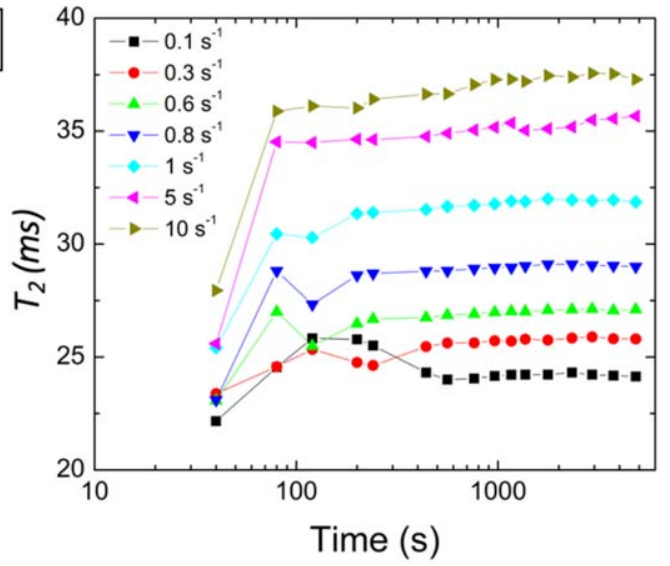

B

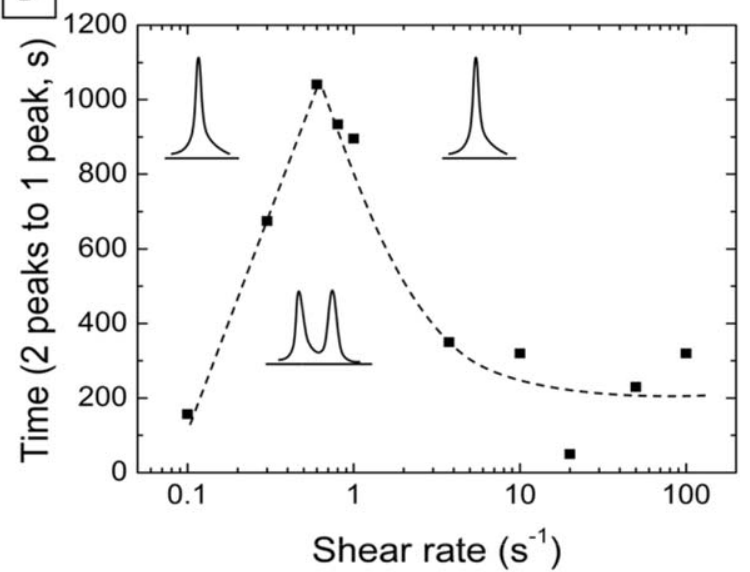

D

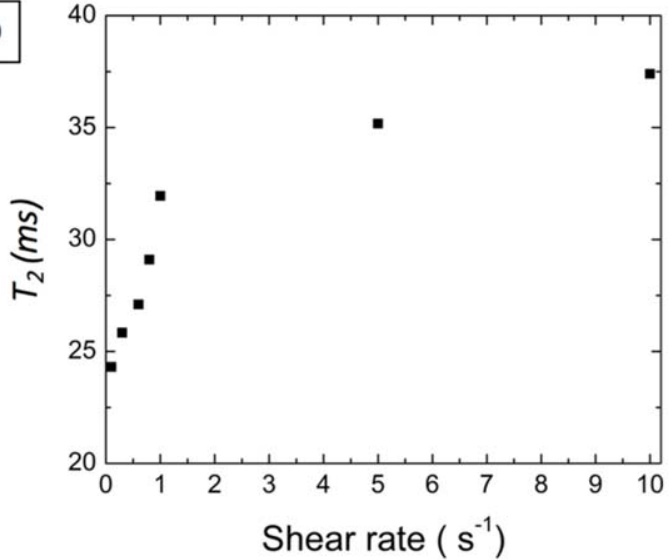

FIGURE 6 (A) Maximum peak-splitting attained for $\mathrm{HPC} / \mathrm{D}_{2} \mathrm{O} 50 \mathrm{wt} \%$ extracted from the deuterium spectra collected during shearing at constant shear rates $\left(0.1-100 \mathrm{~s}^{-1}\right) .^{20,22,32}$ (B) Minimum rest time necessary for the $\mathrm{HPC} / \mathrm{D}_{2} \mathrm{O} 50$ wt $\%$ chiral structure to recover (from peak splitting to a single peak), extracted from the deuterium spectra collected after cessation of shear at constant shear rates $\left(0.1-100 \mathrm{~s}^{-1}\right) .^{20,22}$ (C) Evolution of the Transversal relaxation time $T_{2}$, with time calculated from the fitting of the magnetization exponential decay function measured at constant shear rates $\left(0.1 \text { to } 10 \mathrm{~s}^{-1}\right)^{22}$ (D) Evolution of $T_{2}$ with shear rate $\left(T_{2}\right.$ values were selected from (C) in the stationary region, at $2000 \mathrm{~s}$ ). [Color figure can be viewed at wileyonlinelibrary.com]

(Fig. 7). With this protocol, we ensure that the recovery of the structure is hampered, as confirmed by Rheo-NMR [Fig. 6(B)].
As observed in Figure 7(A), pre-shearing the sample with such protocol does not affect the viscosity as it overlays the results of the viscosity values obtained without any pre-

TABLE 1 Summary of the Different Regimes Encountered in All Shear Rates

\begin{tabular}{|c|c|c|c|c|}
\hline & Region I & Region II & Region III & \\
\hline$\dot{\gamma}\left[\mathrm{s}^{-1}\right]$ & $0.01-0.06$ & $0.06-1$ & $1-15$ & $>15$ \\
\hline Behavior & $\begin{array}{l}\text { Disordered } \\
\text { cholesteric } \\
\text { domains. }^{10}\end{array}$ & $\begin{array}{l}\text { Cholesteric domains } \\
\text { alignment }\end{array}$ & Helix Uncoiling & $\begin{array}{l}\text { Dynamics of the } \\
\text { pseudo-nematic phase. }\end{array}$ \\
\hline$N_{1}$ & \multicolumn{2}{|c|}{$\begin{array}{c}\text { No dependency with shear } \\
\text { (positive values but close to zero) }\end{array}$} & $\begin{array}{l}\text { First minimum of } \\
N_{1} \sim 3-4 s^{-1}\end{array}$ & $\begin{array}{l}\text { Seond minimum of } \\
N_{1} \sim 40 \mathrm{~s}^{-1}\end{array}$ \\
\hline Rheo-NMR & & $\begin{array}{l}\text { Tumbling regime of } \\
\text { the helices. } \\
\text { Relaxation time } \\
\text { increases with pre-shear }\end{array}$ & $\begin{array}{l}\text { Quadrupolar splitting evolved } \\
\text { toward a maximum value. } \\
\text { Relaxation time increases } \\
\text { with pre-shear }\end{array}$ & $\begin{array}{l}\text { Maximum degree of } \\
\text { order is achieved at } 15-20 \mathrm{~s}^{-1} \text {. } \\
\text { Relaxation time decreases } \\
\text { with pre-shear }\end{array}$ \\
\hline $\begin{array}{l}\text { Theory (Helix in } \\
\text { vorticity direction) }\end{array}$ & $\begin{array}{l}\lambda<1 \text { with } \\
\operatorname{low} D_{\mathrm{e}}\end{array}$ & $\begin{array}{l}\text { Oscillations of } \lambda \\
\text { between } \lambda_{\min } \text { and } \lambda_{\max }\end{array}$ & $\begin{array}{l}\dot{\gamma}_{c}=1.77 \mathrm{~s}^{-1} \\
\text { For helix uncoiling }\end{array}$ & \\
\hline
\end{tabular}



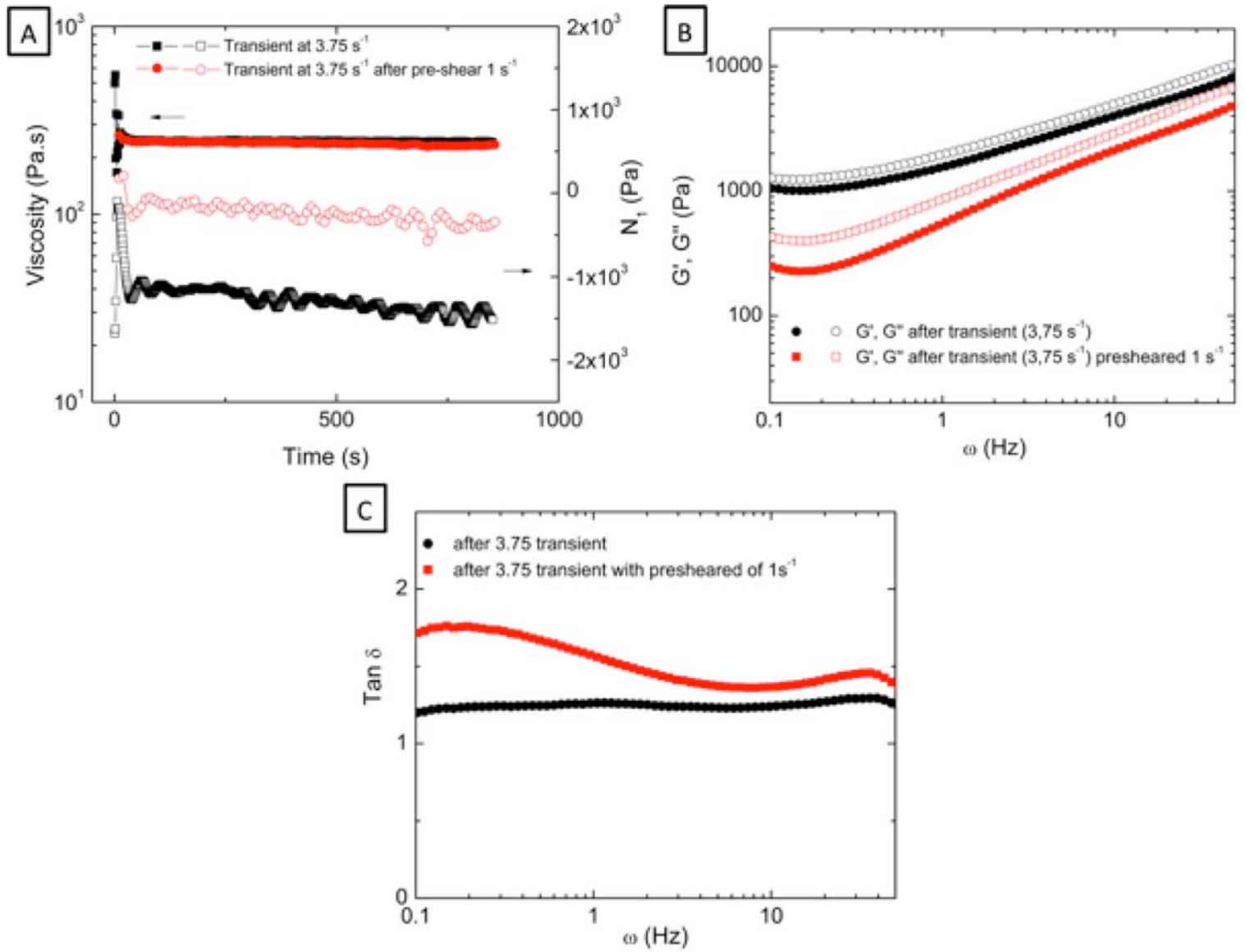

FIGURE 7 (A) Transient measurement performed for LC-HPC at shear rate of $3.75 \mathrm{~s}^{-1}$ (squares) and with a pre-shear of $1 \mathrm{~s}^{-1}$ (circles). Frequency sweep tests performed immediately after transient measurements in the linear viscoelastic regime (LVR, constant strain of $0.01 \%$ ) in which (B) $G^{\prime}$ and $G^{\prime \prime}$ and (C) $\tan \delta$ are represented. [Color figure can be viewed at wileyonlinelibrary.com]

shear treatment. But, its effect in the $N_{1}$ behavior is evident as it becomes less negative with the application of the preshear. The almost suppression of this first minimum of $N_{1}$ (also observed in Supporting Information Fig S1A) could be related to the induced orientation and possible deformation or even uncoiling of the helix.

As it is known, normal forces such as $N_{1}$ provide information regarding the stored elastic energy during flow. ${ }^{17}$ Following that, with the aim of understand the phenomenology behind the first minimum of $N_{1}$, we performed frequency sweep tests to the LC-HPC sample immediately after both transient measurements tests (with and without pre-shear treatments). As observed from Figure 7(B), where the evolution with frequency of elastic modulus $\left(G^{\prime}\right)$ and viscous modulus $\left(G^{\prime \prime}\right)$ are plotted, the LC-HPC sample presents a liquid-like behavior being $G^{\prime \prime}$ higher than $G^{\prime}$ in all the frequency range. However, $G^{\prime}$ and $G^{\prime \prime}$ both decrease almost one order of magnitude when LC-HPC is pre-sheared. These reductions in the viscoelastic properties are accompanied by an increase of loss tangent $\left(\tan \delta=G^{\prime \prime} / G^{\prime}\right)$, which indicates that the presheared sample have a more fluid-like character. As a consequence of pre-shear treatment, the LC-HPC sample gives rise to a decrease of the viscoelastic properties, which may be correlated to a less structured system due to shear-induced orientation and further uncoiling of the cholesteric helix.

\section{CONCLUSIONS}

This study presents an experimental evidence of the theoretically predicted shear-induced helix uncoiling. In fact, in this work, for the first time a shear rate regime where this transition may occur is determined by standard rheology. The detection of this helix uncoiling was performed either by the construction of a flow curve from data obtained under steady state, as well as from a shear rate ramp measurement where the system was certainly not under steady state conditions.

Therefore, considering the obtained experimental results, together with the conclusions already found in the literature, we could picture the shear rate dependence of the cholesteric LC-HPC as follows:

At low shear rates, the cholesteric domains flow over each other in a disordered environment deriving in the first shear thinning of the viscosity as stated by Onogi and Asada. When the pseudo-Newtonian plateau is achieved and $N_{1}$ shows an 
independent behavior to the applied shear, cholesteric domains of LC are aligned in the flow direction. As the shear rate increases, (between 1 and $15 \mathrm{~s}^{-1}$ ) the first minimum of $N_{1}$ is observed. We consider that this could be the shear rate range where the helix uncoiling occurs. This minimum could be correlated to the loss of stored energy and further elastic change in the LC-HCP system consequent from the cholesteric helix uncoiling. The absence of this $N_{1}$ minimum in the Sigillo et al. and Grizutti et al. results shown in Figure 1 is puzzling, but most probably it is linked with the initial strain applied to the sample at each shear rate that may have suppressed the cholesteric structure. As the shear rate increases, an inflexion point found in between the two minima of $N_{1}$ is observed at $15 \mathrm{~s}^{-1}$. This point delimits the shear rate range above which the non-equilibrium nematic monodomain is encountered. Beyond this shear rate, the LC-HPC presents the behavior already described in the literature. Moreover, we consider that at $15 \mathrm{~s}^{-1}$ a tumbling-wagging process of the nematic director sets in, according to the literature. As the shear rate increases, the $N_{1}$ exhibits a decrease toward a second negative minimum of $N_{1}$. Only polymeric nematic LCs possess a molecular elasticity associated with flow-induced distortions of the degree of molecular alignment or order parameter $^{33}$ and this distortion is represented as a secondary minimum of $N_{1}$ in the flow curve. The wagging of the nematic director induces this second $N_{1}$ minimum. ${ }^{11,34,35}$ Finally, at shear rates above $45 \mathrm{~s}^{-1}$ the linear increment of $N_{1}$ to positive values indicates the alignment of the nematic director. In fact, as determined by Rheo-NMR experiments, there is a maximum degree of order that is achieved at a crictial shear rates of $15-20 \mathrm{~s}^{-1}$. Above this shear rate, the alignment of the director is complete and independent of the shear.

\section{ACKNOWLEDGMENTS}

This work is also funded by FEDER funds through the COMPETE 2020 Programme and National Funds through FCTPortuguese Foundation for Science and Technology under projects POCI-01-0145-FEDER-007688 (Reference UID/CTM/ 50025), PTDC/FIS-NAN/0117/2014, PTDC/CTM-BIO/6178/ 2014 and the Portuguese NMR Network RNRMN "Rede Nacional de RMN." C. Echeverria acknowledges the Ministry of Science, Technology and Higher Education for National Funds, European Social Funds and FCT for fellowship number SFRH/ BPD/88779/2012. A. D. Rey is grateful for financial support from the Natural Sciences and Engineering Council of Canada.

\section{REFERENCES AND NOTES}

1 H. Siebert, D. Grabowski, C. Schmidt, Rheol. Acta 1997, 36, 618-627.

2 R. G. Larson, L. A. Archer, Liq. Cryst. 1995, 19, 883-885.

3 K. Hongladarom, W. R. Burghardt, Rheol. Acta 1998, 37, 46-53.

4 W. R. Burghardt, G. G. Fuller, Macromolecules 1991, 24, 2546-2555.
5 P. L. Maffettone, G. Marrucci, M. Mortier, P. Moldenaers, J. Mewis, J. Chem. Phys. 1994, 100, 7736-7743.

6 K. Hongladarom, V. Secakusuma, W. R. Burghardt, J. Rheol. (1978-Present) 1994, 38, 1505-1523.

7 K. F. Wissbrun, J. Rheol. (1978-Present) 1981, 25, 619-662.

$8 \mathrm{~S}$. Onogi, T. Asada, Rheology and Rheo-Optics of Polymer Liquid Crystals. In G. Astarita, G. Marrucci, L. Nicolais, Eds.; Rheology, Springer: US, 1980; pp 127-147.

9 C. Macosko, Principle, Measurements, and Applications, Rheology. Wiley-VCH: New York, 1994.

10 R. Byron Bird, R. Armstrong, O. Hassager, Dynamics of Polymeric Liquids. Fluid Mechanics, Wiley: New York, 1977.

11 R. G. Larson, Macromolecules 1990, 23, 3983-3992.

12 C. M. Huang, J. J. Magda, R. G. Larson, J. Rheol. 1999, 43, 31-50.

13 G. Marrucci, P. L. Maffettone, Macromolecules 1989, 22, 4076-4082.

14 I. Sigillo, N. Grizzuti, J. Rheol. (1978-Present) 1994, 38, 589599.

15 A. D. Rey, Phys. Rev. E 1996, 53, 4198-4201.

16 A. D. Rey, J. Nonnewton. Fluid Mech. 1996, 64, 207-227.

17 S. B. Kharchenko, J. F. Douglas, J. Obrzut, E. A. Grulke, K. B. Migler, Nat. Mater. 2004, 3, 564-568.

18 Y. Geng, P. L. Almeida, S. N. Fernandes, C. Cheng, P. PalffyMuhoray, M. H. Godinho,. Sci. Rep. 2013, 3, 1028.

19 C. Echeverria, L. E. Aguirre, E. G. Merino, P. L. Almeida, M. H. Godinho, ACS Appl. Mater. Interfaces 2015, 7, 21005-21009.

20 C. Echeverria, P. L. Almeida, G. Feio, J. L. Figueirinhas, A. D. Rey, M. H. Godinho, Polymer 2015, 65, 18-25.

21 C. Echeverria, P. L. Almeida, G. Feio, J. L. Figueirinhas, M. H. Godinho, Eur. Polym. J. 2015, 72, 72-81.

22 C. Echeverria, S. N. Fernandes, P. L. Almeida, M. H. Godinho, Eur. Polym. J. 2016, 84, 675-684.

23 N. Grizzuti, P. Moldenaers, M. Mortier, J. Mewis, Rheol. Acta 1993, 32, 218-226.

24 B. Ernst, P. Navard, Macromolecules 1989, 22, 1419-1422.

25 N. Grizzuti, S. Cavella, P. Cicarelli, J. Rheol. 1990, 34, 12931310.

26 G. Kiss, R. S. Porter, J. Polym. Sci.: Polym. Phys. Ed. 1980, 18, 361-388.

27 G. Marrucci, P. L. Maffettone, J. Rheol. (1978-Present) 1990, 34, 1231-1244.

28 G. Marrucci, P. L. Maffettone, J. Rheol. (1978-Present) 1990, $34,1217-1230$.

29 S. G. Baek, J. J. Magda, R. G. Larson, S. D. Hudson, J. Rheol. (1978-Present) 1994, 38, 1473-1503.

30 Y. Farhoudi, A. D. Rey, J. Rheol. (1978-Present) 1993, 37, 289-314.

31 T. Tsuji, A. D. Rey, J. Nonnewton. Fluid Mech. 1997, 73, 127-152.

32 Y. Geng, P. L. Almeida, G. M. Feio, J. L. Figueirinhas, M. H. Godinho, Macromolecules 2013, 46, 4296-4302.

33 R. G. Larson, H. C. Ottinger, Macromolecules 1991, 24, 6270-6282.

34 V. Faraoni, M. Grosso, S. Crescitelli, P. L. Maffettone, J. Rheol. (1978-Present) 1999, 43, 829-843.

35 M. Grosso, S. Crescitelli, E. Somma, J. Vermant, P. Moldenaers, P. L. Maffettone, Phys. Rev. Lett. 2003, 90, 098304. 